\title{
THE THEORY OF AN INSCRIBABLE QUADRILATERAL AND A CIRCLE THAT FORMS PASCAL POINTS
}

\section{DAVID FRAIVERT}

Department of Mathematics

Shaanan College

P.O. Box 906, Haifa 26109

Israel

e-mail: davidfraivert@gmail.com

\begin{abstract}
The theory of a convex quadrilateral and a circle that forms Pascal points is a new topic in Euclidean geometry. The theory deals with the properties of the Pascal points on the sides of a convex quadrilateral, the properties of "circles that form Pascal points", and the special properties of "the circle coordinated with the Pascal points formed by it".

In the present paper, we shall continue developing the theory and expand it to the case where the quadrilateral is inscribable. We prove five new theorems that describe properties in the following subjects:
\end{abstract}

- Necessary and sufficient conditions for a quadrilateral to be inscribable, which are determined by a circle coordinated with the Pascal points formed by it.

- Properties of the perimeters and areas of quadrilaterals inscribed in an inscribable quadrilateral, and that are associated to circles "that form Pascal points on the sides of the quadrilateral".

2010 Mathematics Subject Classification: Primary 51M04, 51M99; Secondary 51M16.

Keywords and phrases: inscribable quadrilateral, tests for the inscribability of a quadrilateral, the method of complex numbers in plane geometry, Pascal points on the sides of a quadrilateral, a circle that forms Pascal points, a circle coordinated with the Pascal points formed by it, Thales' tehorem.

Received November 27, 2016

(C) 2016 Scientific Advances Publishers 


\section{Introduction: General Concepts and Theorems of the Theory of a Convex Quadrilateral and a Circle that Forms Pascal Points}

The theory of a convex quadrilateral and a circle that forms Pascal points deals with a convex quadrilateral $A B C D$ in which the diagonals intersect at point $E$, and the extensions of sides $B C$ and $A D$ intersect at point $F$. In addition, there is a circle $\omega$ that satisfies the following two requirements:

(I) it passes through points $E$ and $F$;

(II) it intersects sides $B C$ and $A D$ at inner points $M$ and $N$, respectively (see Figure 1).

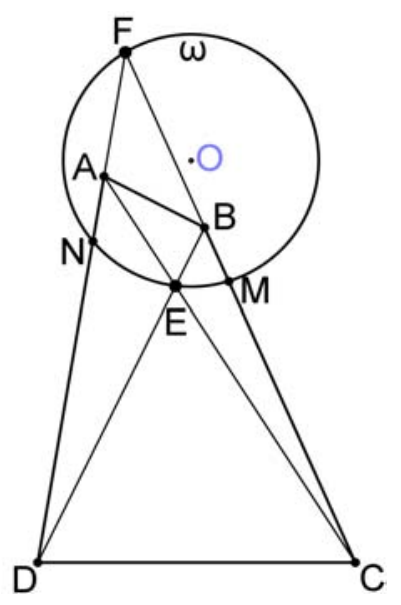

Figure 1.

In this case, the Fundamental Theorem of the theory holds (see [2]):

\section{The Fundamental Theorem.}

Let there be: a convex quadrilateral; a circle that intersects a pair of opposite sides of the quadrilateral, that passes through the point of intersection of the continuations of these sides, and that passes through the point of intersection of the diagonals. 
In addition, let there be four straight lines, each of which passes both through the point of intersection of the circle with a side of the quadrilateral and through the point of intersection of the circle with the continuation of a diagonal.

Then there holds: the straight lines intersect at two points that are located on the other pair of opposite sides of the quadrilateral.

Or, by notation (see Figure 2):

Given: Convex quadrilateral $A B C D$, in which $E=A C \cap B D$, $F=B C \cap A D . \quad$ Circle $\omega$ that satisfies $\quad E, F \in \omega ; M=\omega \cap[B C]$; $N=\omega \cap[A D] ; K=\omega \cap B D ; L=\omega \cap A C$.

Prove that: $K N \cap L M=P \in[A B] ; K M \cap L N=Q \in[C D]$.

We prove the Fundamental Theorem using the general Pascal Theorem (see [2], [3]).

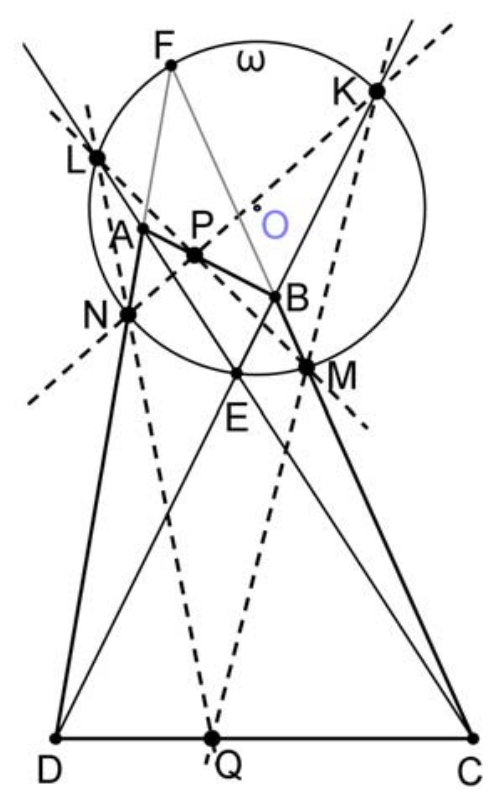

Figure 2. 


\section{Definitions.}

Since the proof of the properties of the points of intersection $P$ and $Q$ is based on Pascal's Theorem:

(I) We shall call these points "Pascal points" on sides $A B$ and $C D$ of the quadrilateral.

(II) We shall call the circle that passes through the points of intersection $E$ and $F$ and through two opposite sides "a circle that forms Pascal points on the sides of the quadrilateral".

Of all the circles that form Pascal points, there is one particular special circle whose center is located on the same straight line together with the Pascal points that are formed by it.

(III) A circle whose center is collinear with the "Pascal points" formed using it will be called: "The circle coordinated with the Pascal points formed by it".

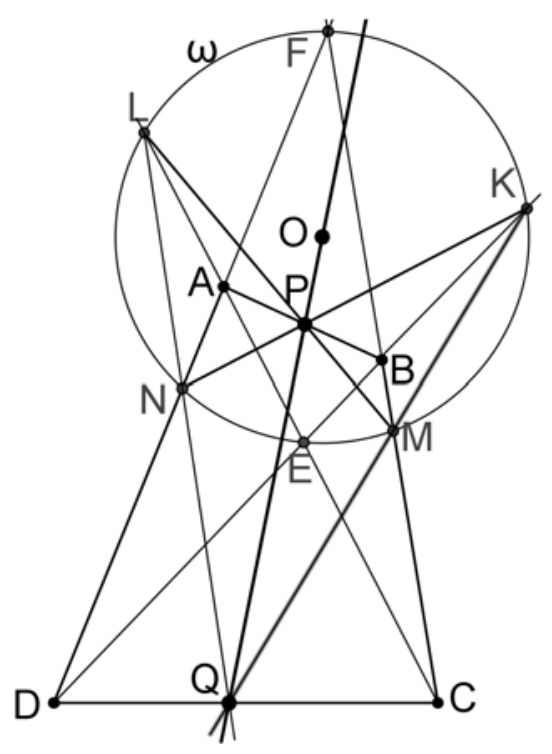

Figure 3. 
For example, in Figure 3, the center of circle $\omega$ (point $O$ ) is collinear with Pascal points $P$ and $Q$, formed using the circle. Therefore, circle $\omega$ is coordinated with the Pascal points formed by it.

We also use the following general theorems of the theory (see proofs in $[2])$ :

\section{General Theorem A.}

Let $\omega_{1}, \omega_{2}$, and $\omega_{3}$ be three circles that pass through sides BC and $A D$ of the quadrilateral, through the point of intersection, $F$, of their continuations, and through the point of intersection, E, of the diagonals.

Then, Pascal points $P_{1}$ and $Q_{1}, P_{2}$ and $Q_{2}, P_{3}$ and $Q_{3}$, which are formed, respectively, using these circles, assign proportional segments on sides $A B$ and $C D$ :

$\frac{P_{1} P_{2}}{P_{2} P_{3}}=\frac{Q_{1} Q_{2}}{Q_{2} Q_{3}}$ (see Figure 4$)$.

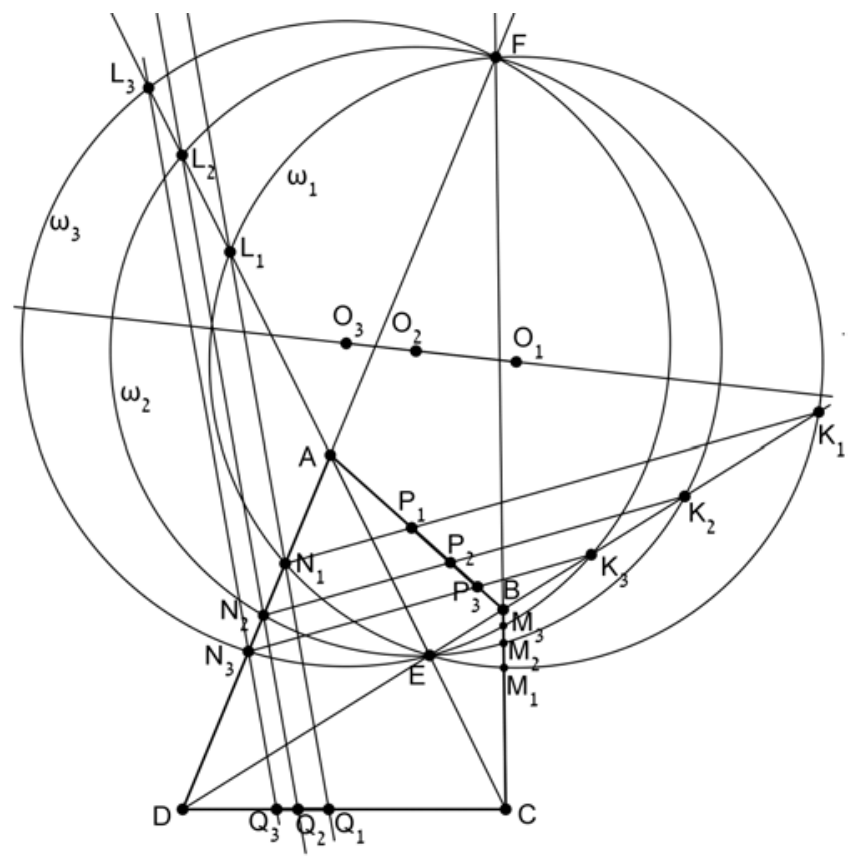

Figure 4 . 
Note: From the proof of General Theorem A, it follows that the corresponding sides of quadrilaterals $P_{1} M_{1} Q_{1} N_{1}$ and $P_{2} M_{2} Q_{2} N_{2}$ are parallel to each other (see Figure 5).

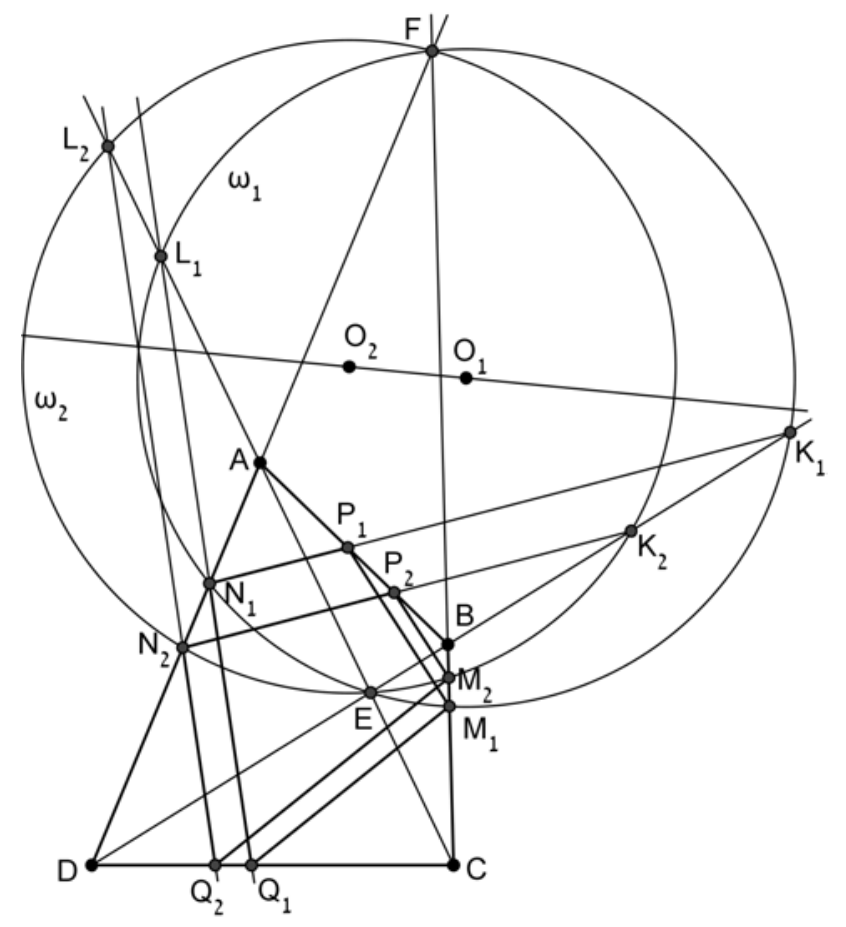

Figure 5.

\section{General Theorem B.}

Let $A B C D$ be a quadrilateral whose diagonals intersect at point $E$, and the continuations of sides $B C$ and $A D$ intersect at point $F$.

And let $\omega$ be a circle that passes through sides $B C$ and $A D$ and through points $E$ and $F ; \omega_{A}-a$ circle that passes through points $A, E$, and $F ; \omega_{B}-a$ circle that passes through points $B, E$, and $F$ (see Figure 6).

Then:

(a) The center of circle $\omega$ (point $O$ ) divides the segment that connects the centers of circles $\omega_{A}$ and $\omega_{B}$ (segment $O_{A} O_{B}$ ) at a ratio that equals 
the ratio at which the Pascal point, $P$, formed using circle $\omega$ divides side $A B$.

(b) In the system in which circle $\omega$ is the unit circle, one can express the ratio $\lambda=\frac{O_{A} O}{O O_{B}}=\frac{A P}{P B}$ using the complex coordinates of points $E, F$, $K, L, M$, and $N$ as follows: $\lambda=\frac{(n-l)(f m-e k)}{(k-m)(f n-e l)}$.

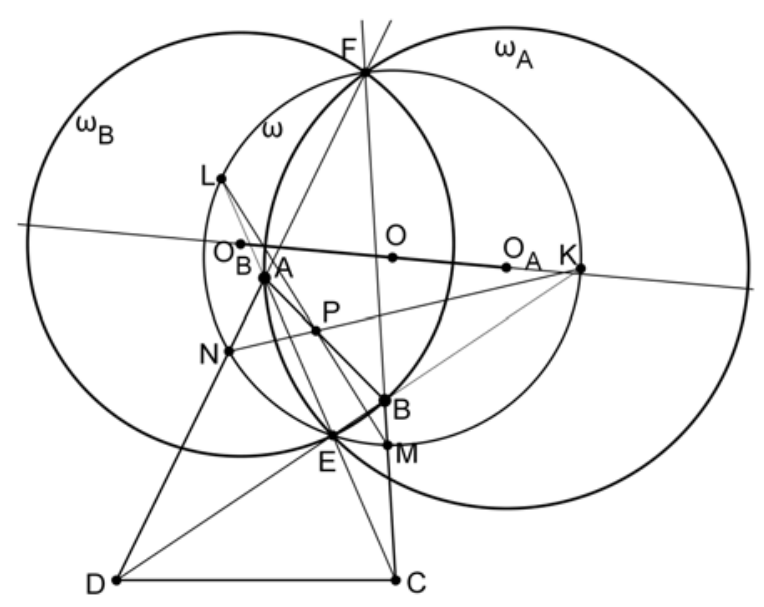

Figure 6.

\section{General Theorem C.}

Let $A B C D$ be a convex quadrilateral, and let $\omega$ be a circle coordinated with the Pascal points $P$ and $Q$ formed by it, where $\omega$ intersects a pair of opposite sides of the quadrilateral at points $M$ and $N$, and also intersects the continuations of the diagonals at points $K$ and $L$ (see Figure 7 ).

Then there holds:

(a) $K L \| M N$;

(b) quadrilateral PMQN is a kite;

(c) in a system in which circle $\omega$ is the unit circle, the complex coordinates of points $K, L, M$, and $N$ satisfy the equality $m n=k l$. 


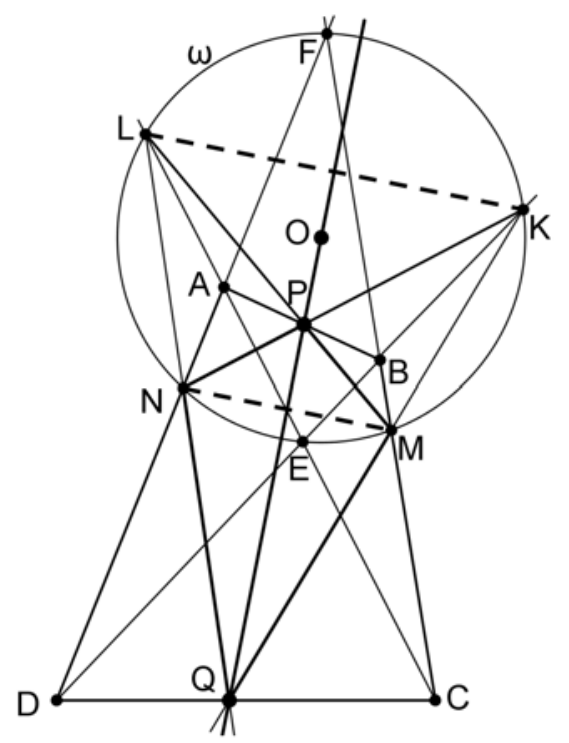

Figure 7.

\section{New Theorems that Hold When the Quadrilateral is Inscribable}

\section{Theorem 1.}

Let $A B C D$ be a quadrilateral in which the diagonals intersect at point $E$, and the continuations of sides $B C$ and $A D$ intersect at point $F$;

$\omega_{E F}$ is a circle with diameter $E F$, and that intersects sides $B C$ and $A D$ at internal points $M$ and $N$, respectively (see Figure 8).

Then quadrilateral $A B C D$ is inscribable iff points $M$ and $N$ divide sides $B C$ and $A D$ at an equal ratio, i.e., there holds $\frac{B M}{M C}=\frac{A N}{N D}$. 


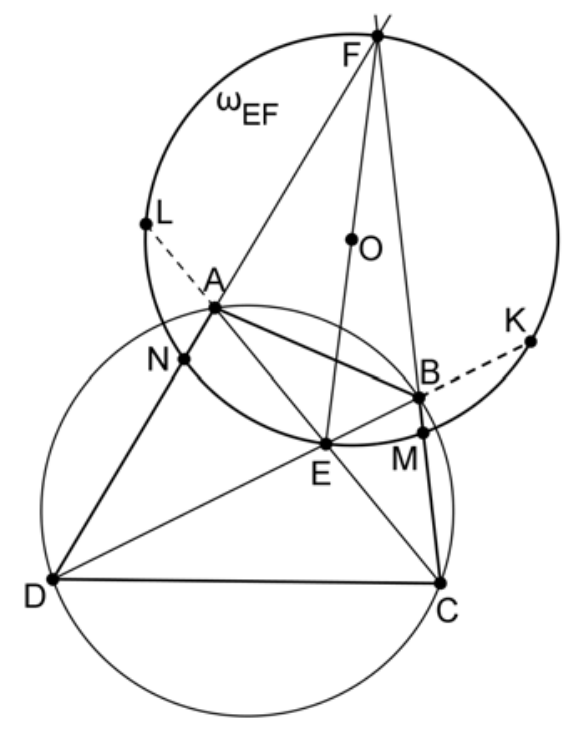

Figure 8.

\section{Proof.}

\section{First direction:}

Given: $A B C D$ is an inscribable quadrilateral, $E=A C \cap B D$ and $F=B C \cap A D ; \quad \omega_{E F}$ is a circle with diameter $E F$, and that satisfies: $M=\omega_{E F} \cap[B C]$ and $N=\omega_{E F} \cap[A D]$.

Prove that: $\frac{B M}{M C}=\frac{A N}{N D}$.

\section{Proof of first direction:}

We extend diagonals $B D$ and $A C$ until they intersect circle $\omega_{E F}$ at the additional points of intersection $K$ and $L$, respectively (see Figure 8). We shall use the method of complex numbers in plane geometry. (The principles of the method and the formulas that we use in the proofs appear, for example, in [5, pp. 154-181].)

We choose a system of coordinates so that circle $\omega_{E F}$ is the unit circle (the center of circle $\omega_{E F}$ is located at the origin (point $O$ ) and the radius is $O E=1$ ). In this system, the equation of the unit circle is 
$z \cdot \bar{z}=1$, where $z$ and $\bar{z}$ are the complex coordinate and its conjugate, for an arbitrary point that is located on circle $\omega_{E F}$. We denote the complex coordinates of points $E, F, K, L, M$, and $N$ by $e, f, k, l, m$, and $n$, respectively. These points are located on the unit circle, and therefore there holds:

$$
\bar{e}=\frac{1}{e}, \bar{f}=\frac{1}{f}, \bar{k}=\frac{1}{k}, \bar{l}=\frac{1}{l}, \bar{m}=\frac{1}{m}, \text { and } \bar{n}=\frac{1}{n} .
$$

We express the complex coordinates (and their conjugates) of points $A, B, C$, and $D$ using the coordinates of the six points that are located on the unit circle.

To this end, we shall make use of the following formulas:

Let $A(a), B(b), C(c)$, and $D(d)$ be four points that belong to the unit circle, and let $S(s)$ be the point of intersection of the straight lines that pass through the chords $A B$ and $C D$ in the unit circle.

For the complex coordinate of $S$ and its conjugate, there holds:

$$
\bar{s}=\frac{a+b-c-d}{a b-c d},
$$

and

$$
s=\frac{b c d+a c d-a b d-a b c}{c d-a b} .
$$

From these formulas, and since $e=-f$ (because segment $E F$ is a diameter of the unit circle), we obtain:

$$
\begin{aligned}
& \bar{a}=\frac{2 f+n-l}{f(n+l)} \text { and } a=\frac{2 n l+f l-f n}{n+l}(\text { because } A=F N \cap E L, \text { see Figure 8); } \\
& \left.\bar{b}=\frac{2 f+m-k}{f(m+k)} \text { and } b=\frac{2 m k+f k-f m}{m+k} \text { (because } B=F M \cap E K\right) ; \\
& \left.\bar{c}=\frac{2 f+m-l}{f(m+l)} \text { and } c=\frac{2 m l+f l-f m}{m+l} \text { (because } C=F M \cap E L\right) ; \\
& \left.\bar{d}=\frac{2 f+n-k}{f(n+k)} \text { and } d=\frac{2 n k+f k-f n}{n+k} \text { (because } D=F N \cap E K\right)
\end{aligned}
$$


Since it is given that quadrilateral $A B C D$ is inscribable, it follows that the complex coordinates of points $A, B, C$, and $D$ satisfy the following equation (see [6, Paragraph 7]):

$$
\frac{a-c}{b-c}: \frac{a-d}{b-d}=\frac{\bar{a}-\bar{c}}{\bar{b}-\bar{c}}: \frac{\bar{a}-\bar{d}}{\bar{b}-\bar{d}}(*)
$$

Let us calculate the two sides of the equality $(*)$ :

We substitute the expressions for $a, b, c$, and $d$ in the left-hand side of equality $(*)$, and obtain:

$$
\begin{aligned}
\frac{a-c}{b-c}: \frac{a-d}{b-d} \\
\quad=\frac{\frac{2 n l+f l-f n}{n+l}-\frac{2 m l+f l-f m}{m+l}}{\frac{2 m k+f k-f m}{m+k}-\frac{2 m l+f l-f m}{m+l}} \times \frac{\frac{2 m k+f k-f m}{m+k}-\frac{2 n k+f k-f n}{n+k}}{\frac{2 n l+f l-f n}{n+l}-\frac{2 n k+f k-f n}{n+k}} .
\end{aligned}
$$

After adding together the fractions and simplifying the numerator and the denominator, we obtain the following expression:

$$
\frac{k l(m-n)^{2}(f-l)(f-k)}{m n(k-l)^{2}(f+m)(f+n)} .
$$

Similarly, we substitute the expressions for $\bar{a}, \bar{b}, \bar{c}$, and $\bar{d}$ in the right-hand side of equality $(*)$, and obtain:

$$
\begin{gathered}
\frac{\bar{a}-\bar{c}}{\bar{b}-\bar{c}}: \frac{\bar{a}-\bar{d}}{\bar{b}-\bar{d}}=\frac{\frac{2 f+n-l}{f(n+l)}-\frac{2 f+m-l}{f(m+l)}}{\frac{2 f+m-k}{f(m+k)}-\frac{2 f+m-l}{f(m+l)}} \times \frac{\frac{2 f+m-k}{f(m+k)}-\frac{2 f+n-k}{f(n+k)}}{\frac{2 f+n-l}{f(n+l)}-\frac{2 f+n-k}{f(n+k)}} \\
=\frac{(f m-m l+n l-f n) \cdot(f n-n k+m k-f m)}{(f l-m k+m l-f k) \cdot(f k-n l+n k-f l)}=\frac{(m-n)^{2}(f-l)(f-k)}{(k-l)^{2}(f+m)(f+n)}
\end{gathered}
$$

Now we substitute the results obtained in the two sides of equality $(*)$, and obtain:

$$
\frac{k l(m-n)^{2}(f-l)(f-k)}{m n(k-l)^{2}(f+m)(f+n)}=\frac{(m-n)^{2}(f-l)(f-k)}{(k-l)^{2}(f+m)(f+n)} .
$$


From this equality, we obtain $\frac{k l}{m n}=1$ or $k l=m n(* *)$.

Now let us consider the ratios $\frac{A N}{N D}$ and $\frac{B M}{M C}$ :

Using the complex coordinates of the points $A, N$, and $D$, the first ratio can be expressed as:

$$
\frac{A N}{N D}=\frac{n-a}{d-n}=\frac{n-\frac{2 n l+f l-f n}{n+l}}{\frac{2 n k+f k-f n}{n+k}-n}=\frac{(n-l)(n+k)}{(k-n)(n+l)} .
$$

In a similar manner, for the second ratio we obtain:

$$
\frac{B M}{M C}=\frac{(m-k)(m+l)}{(l-m)(m+k)} .
$$

Now let us consider the difference of the ratios:

$$
\frac{A N}{N D}-\frac{B M}{M C}=\frac{(n-l)(n+k)}{(k-n)(n+l)}-\frac{(m-k)(m+l)}{(l-m)(m+k)} .
$$

After adding the fractions and simplifying the numerator, we obtain:

$$
\frac{2 l m^{2} n-2 k m^{2} n+2 k^{2} l m-2 k l^{2} m+2 l m n^{2}-2 k l^{2} n+2 k^{2} l n}{(k-n)(n+l)(l-m)(m+k)} .
$$

Since the complex coordinates of points $K, L, M$, and $N$ satisfy the relation $k l=m n$, the last fraction can be converted into:

$$
\begin{array}{r}
\frac{2 m n(l m-k m+k m-k n-l m+l n-l n+k n)}{(k-n)(n+l)(l-m)(m+k)} \\
=\frac{2 m n \cdot 0}{(k-n)(n+l)(l-m)(m+k)}=0 .
\end{array}
$$

We obtained that the ratio difference equals 0 , therefore there holds: $\frac{A N}{N D}=\frac{B M}{M C}$. 


\section{Second direction:}

Given: $A B C D$ is a quadrilateral in which, $E=A C \cap B D$ and $F=B C \cap A D ; \omega_{E F}$ is a circle with diameter $E F$, and that satisfies:

$M=\omega_{E F} \cap[B C]$ and $N=\omega_{E F} \cap[A D] ; \frac{B M}{M C}=\frac{A N}{N D}$.

Prove that: Quadrilateral $A B C D$ is inscribable in a circle.

\section{Proof of the second direction:}

In proving the first direction, we obtained the following expressions for the ratios $\frac{A N}{N D}$ and $\frac{B M}{M C}$ :

$$
\frac{A N}{N D}=\frac{(n-l)(n+k)}{(k-n)(n+l)} \text { and } \frac{B M}{M C}=\frac{(m-k)(m+l)}{(l-m)(m+k)} .
$$

Therefore, the following relation holds:

$$
\frac{(n-l)(n+k)}{(k-n)(n+l)}-\frac{(m-k)(m+l)}{(l-m)(m+k)}=0
$$

The left-hand side of the equality can be brought to the following form:

$$
\frac{l m n^{2}-k m n^{2}+k^{2} l n-k m^{2} n-k l^{2} n+l m^{2} n-k l^{2} m+k^{2} l m}{(k-n)(n+l)(l-m)(m+k)}=0,
$$

and hence, after factoring the numerator, we obtain the following equation:

$$
(l-k)(m+n)(m n-k l)=0 .
$$

Since $l \neq k$ and $m \neq-n$ (the segment $M N$ is not a diameter of the unit circle), from the last equation it follows that $m n-k l=0$, or $m n=k l$. 
We now return to the expressions $\frac{a-c}{b-c}: \frac{a-d}{b-d}$ and $\frac{\bar{a}-\bar{c}}{\bar{b}-\bar{c}}: \frac{\bar{a}-\bar{d}}{\bar{b}-\bar{d}}$. In proving the first direction, we saw that these expressions equal the expressions $\frac{k l(m-n)^{2}(f-l)(f-k)}{m n(k-l)^{2}(f+m)(f+n)}$ and $\frac{(m-n)^{2}(f-l)(f-k)}{(k-l)^{2}(f+m)(f+n)}$, respectively. We can reduce the first fraction by $m n=k l$, therefore the expressions are identical. It thus follows that the complex coordinates of points $A, B, C$, and $D$ satisfy the equality (*).

Therefore, quadrilateral $A B C D$ is inscribable.

\section{Theorem 2.}

Let $A B C D$ be a quadrilateral in which the diagonals intersect at point $E$, and the continuations of sides $B C$ and $A D$ intersect at point $F$; $\omega_{E F}$ is a circle with the diameter EF, and that forms Pascal points $P$ and $Q$ on sides $A B$ and $C D$, respectively.

Then quadrilateral $A B C D$ is inscribable iff $\omega_{E F}$ is a circle that is coordinated with the Pascal points formed by it (points $P$ and $Q$ are collinear with center $O$ of circle $\omega_{E F}$, as shown in Figure 9).

\section{Proof.}

\section{First direction:}

Given: $A B C D$ is an inscribable quadrilateral, $E=A C \cap B D$ and $F=B C \cap A D ; \quad \omega_{E F}$ is a circle with diameter $E F$, and that satisfies: $M=\omega_{E F} \cap[B C], N=\omega_{E F} \cap[A D], K=\omega_{E F} \cap B D$, and $L=\omega_{E F} \cap A C$.

Prove that: The center of circle $\omega_{E F}$ (point $O$ ), and Pascal points $P$ and $Q$ formed by it lie on the same straight line. 


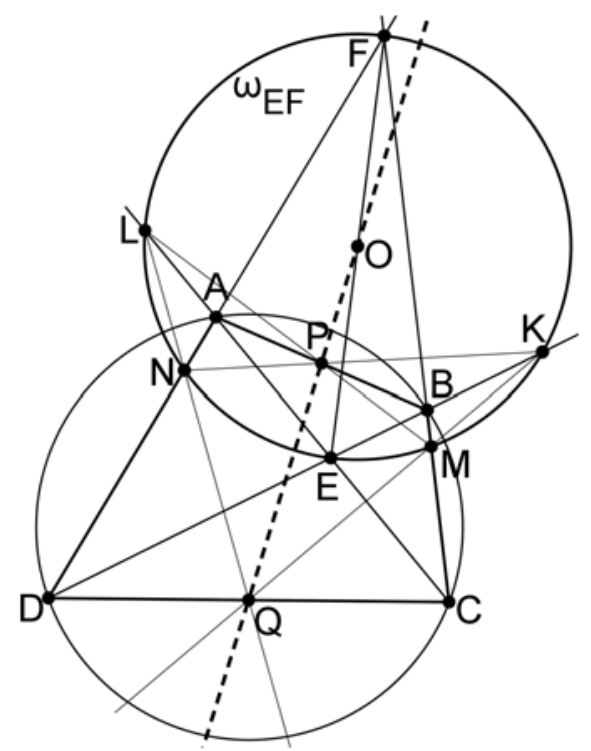

Figure 9.

\section{Proof of first direction:}

We select a system of coordinates such that circle $\omega_{E F}$ is the unit circle ( $O$ is the origin and the radius is $O E=1$ ). We express the complex coordinates of Pascal points $P$ and $Q$ (and their conjugates) through the coordinates of points $M, N, K$, and $L$ (the points of intersection of $\omega_{E F}$ with sides $B C$ and $A D$, and with the continuations of diagonals $B D$ and $A C)$.

We use formulas (i) and (ii) that were presented in the proof of the first direction of Theorem 1, and the fact that $P=K N \cap M L$ and $Q=K M \cap L N$, and obtain:

$$
\begin{aligned}
& \bar{p}=\frac{n+k-m-l}{n k-m l} \text { and } p=\frac{m k l+m n l-n k l-m n k}{m l-n k}, \\
& \bar{q}=\frac{m+k-n-l}{m k-n l} \text { and } q=\frac{n k l+m n l-m k l-m n k}{n l-m k} .
\end{aligned}
$$


In addition, in the proof of Theorem 1, we saw that for an inscribable quadrilateral $A B C D$ and a unit circle $\omega_{E F}$, there holds the equality $m n=k l$, where $k, l, m$, and $n$ are the complex coordinates of intersection points $K, L, M$, and $N$.

Therefore, the expressions for $p$ and $q$ can be written down in a simpler form:

$$
p=\frac{m n(m+l-n-k)}{m l-n k} \text { and } q=\frac{m n(n+l-m-k)}{n l-m k} .
$$

The collinear property of points $P, Q$ and origin $O$ is equivalent to the satisfaction of the equality $p \bar{q}-q \bar{p}=0(*)$.

Let us consider the difference $p \bar{q}-q \bar{p}$. We substitute the expressions for $p, \bar{p}, q$, and $\bar{q}$, and obtain:

$$
\begin{aligned}
p \bar{q}-q \bar{p}=\frac{m n(m+l-n-k)}{m l-n k} \cdot \frac{m+k-n-l}{m k-n l} & \\
& \quad-\frac{m n(n+l-m-k)}{n l-m k} \cdot \frac{n+k-m-l}{n k-m l}=0 .
\end{aligned}
$$

Therefore, points $P$ and $Q$ are collinear with the point $O$.

\section{Second direction:}

Given: $A B C D$ is a quadrilateral, $E=A C \cap B D$ and $F=B C \cap A D$; $\omega_{E F}$ is a circle (whose center is at point $O$ and whose diameter is the segment $E F)$, which satisfies: $M=\omega_{E F} \cap[B C], N=\omega_{E F} \cap[A D]$, $K=\omega_{E F} \cap B D$, and $L=\omega_{E F} \cap A C$; the point $O$ and the Pascal points $P$ and $Q$ that are formed by $\omega_{E F}$ lie on the same straight line.

Prove that: Quadrilateral $A B C D$ is inscribable.

\section{Proof of the second direction:}

From the data of the second direction, it follows that $\omega_{E F}$ is a circle that is coordinated with the Pascal points formed by it. Therefore, based on General Theorem C (Section c) which we stated in the Introduction, 
the complex coordinates of points $K, L, M$, and $N$ satisfy the equality $k l=m n$. Hence, in a manner similar to the proof of the second direction of Theorem 1, we prove that quadrilateral $A B C D$ is inscribable.

\section{Note:}

Theorems 1 and 2 (the second directions thereof) suggest the following two tests for checking the inscribability of a quadrilateral:

A quadrilateral $A B C D$ is inscribable if for a circle whose diameter is the segment that connects the point of intersection of the diagonal and the point of intersection of the continuation of two opposite sides there holds:

(1) The two points of intersection of the circle with the two opposite sides divide them by an equal ratio (Theorem 1).

(2) The circle is the circle coordinated with the Pascal points formed by it (Theorem 2).

\section{Theorem 3.}

Given is an inscribable quadrilateral, and a circle whose diameter is the segment that connects the point of intersection of the diagonals and the point of intersection of the continuations of the two opposite sides.

Then, the Pascal point formed by this circle is the middle of the two other opposite sides in the quadrilateral.

Given: $A B C D$ is an inscribable quadrilateral, $E=A C \cap B D$ and $F=B C \cap A D ; \quad \omega_{E F}$ is a circle with diameter $E F$, and that satisfies: $M=\omega_{E F} \cap[B C], N=\omega_{E F} \cap[A D], K=\omega_{E F} \cap B D$, and $L=\omega_{E F} \cap A C$.

Prove that: Pascal points $P$ and $Q$ that are formed by $\omega_{E F}$ are the middles of sides $A B$ and $C D$, respectively. 


\section{Proof.}

We denote the centers of circles $\omega_{A}$ and $\omega_{B}$ that pass through points $A, E, F$ and $B, E, F$, respectively, by $O_{A}$ and $O_{B}$, respectively (see Figure 10).

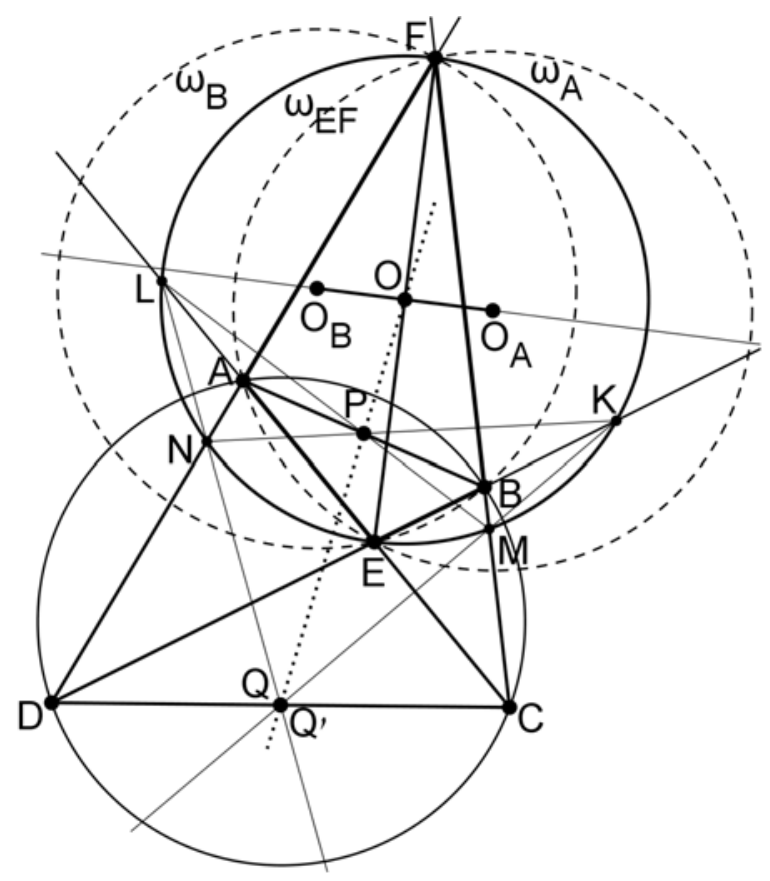

Figure 10.

From General Theorem B that is presented in the introduction, it follows that the center of circle $\omega_{E F}$ (point $O$ ) divides segment $O_{A} O_{B}$ by a ratio that is equal to the ratio by which the Pascal point $P$ divides segment $A B$.

In the coordinate system in which circle $\omega_{E F}$ is the unit circle $(O$ is the origin, and the radius is $O E=1)$, one can express this ratio as: $\lambda=\frac{(n-l)(f m-e k)}{(k-m)(f n-e l)}$, where $e, f, k, l, m$, and $n$ are the complex coordinates of points $E, F, K, L, M$, and $N$, respectively.

Let us prove that point $O$ is the middle of segment $O_{A} O_{B}$. 
We calculate the ratio $\lambda=\frac{O_{A} O}{O O_{B}}$, by which point $O$ divides segment $O_{A} O_{B}$. Since segment $E F$ is a diameter in the unit circle and the quadrilateral $A B C D$ is inscribable, it follows that the following two equalities hold: $f=-e$, and $k l=m n$.

Therefore,

$$
\begin{aligned}
\lambda & =\frac{(n-l)(f m-e k)}{(k-m)(f n-e l)}=\frac{(n-l)(f m+f k)}{(k-m)(f n+f l)} \\
& =\frac{m n+n k-m l-k l}{n k+k l-m n-m l}=\frac{n k-m l}{n k-m l}=1,
\end{aligned}
$$

and therefore point $O$ is the middle of the segment $O_{A} O_{B}$. It follows from General Theorem $\mathrm{B}$, that $P$ is the middle of segment $A B$.

Pascal point $Q$ is known to belong to side $C D$.

We mark by $Q^{\prime}$ the middle of segment $C D$. We prove that point $Q^{\prime}$ is collinear with points $P$ (the middle of $A B$ ) and $O$ (the middle of $E F$ ). We use the following property of the complete quadrilateral (see [4, Section 194]: "in the complete quadrilateral, the middles of the diagonals are on the same straight line".

In our case, in the complete quadrilateral $A F B E C D$ (see Figure 10), points $P, Q^{\prime}$, and $O$ are the middles of diagonals $A B, C D$, and $E F$, respectively. Therefore, they are on the same straight line.

On the other hand, from Theorem 2 (the first direction), also point $Q$ is collinear with points $P$ and $O$. Therefore, it necessarily follows that points $Q$ and $Q^{\prime}$ coincide. We obtain that $Q$ is the middle of side $C D$.

\section{Conclusion from Theorems 2 and 3}

(Another (third) test for the inscribability of a quadrilateral.)

In a quadrilateral in which $E$ is the point of intersection of the diagonals, and $F$ is the point of intersection of the continuations of two opposite sides, there holds the following: If the circle in which segment EF 
is a diameter is a "circle that forms Pascal points on the sides of the quadrilateral', and in addition, these Pascal points are the middles of the sides, then the quadrilateral is inscribable.

\section{Theorem 4.}

Let $A B C D$ be an inscribable quadrilateral in which the diagonals intersect at point $E$, and the continuations of sides $B C$ and $A D$ intersect at point $F$;

$\omega_{i}$ is an arbitrary circle that intersects sides $B C$ and $A D$ at points $M_{i}$ and $N_{i}$, respectively, and which passes through points $E$ and $F$;

$P_{i}$ and $Q_{i}$ are the Pascal points formed by $\omega_{i}$ on sides $A B$ and $C D$, respectively.

Then, for any such circle $\omega_{i}$, the perimeter of quadrilateral $P_{i} M_{i} Q_{i} N_{i}$ is a fixed value that does not depend on the choice of $\omega_{i}$.

\section{Proof.}

It is sufficient to prove that the perimeter of an arbitrary quadrilateral $P_{i} M_{i} Q_{i} N_{i}$ equals the perimeter of the quadrilateral $P M Q N$ defined by means of circle $\omega_{E F}$ (whose diameter is $E F$ ).

From Theorem 3, Pascal points $P$ and $Q$ are the middles of sides $A B$ and $C D$, respectively (see Figure 11). 


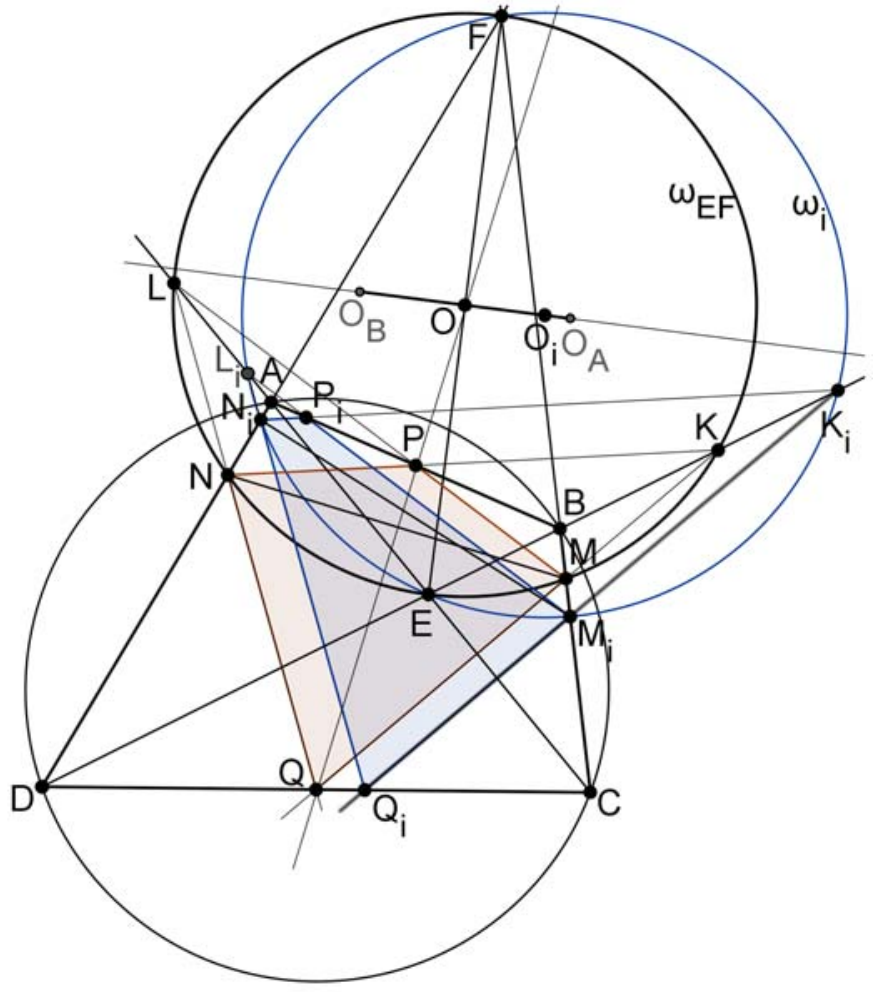

Figure 11.

From the proof of General Theorem A (see [2, Theorem 2]), it follows that the corresponding sides of quadrilaterals $P_{i} M_{i} Q_{i} N_{i}$ and $P M Q N$ are parallel. Therefore, from the extended Thales' theorem, and from the fact that $N_{i} P_{i} \| N P$, it follows that $\frac{A N_{i}}{A N}=\frac{A P_{i}}{A P}=\frac{N_{i} P_{i}}{N P}$ we denote $\alpha$.

Hence, it follows that $A P_{i}=\alpha \cdot A P$ and that $N_{i} P_{i}=\alpha \cdot N P$.

In a similar manner, since $M_{i} P_{i} \| M P$, we obtain that:

$\frac{B M}{B M_{i}}=\frac{B P}{B P_{i}}=\frac{P M}{P_{i} M_{i}}$ we denote $\beta$, and hence $B P_{i}=\frac{B P}{\beta}$ and $P_{i} M_{i}=\frac{P M}{\beta}$.

From $M_{i} Q_{i} \| M Q$, we obtain $\frac{C M_{i}}{C M}=\frac{C Q_{i}}{C Q}=\frac{M_{i} Q_{i}}{M Q}$ we denote $\gamma$, 
and hence $C Q_{i}=\gamma \cdot C Q$ and $M_{i} Q_{i}=\gamma \cdot M Q$.

From $N_{i} Q_{i} \| N Q$, we obtain $\frac{D Q}{D Q_{i}}=\frac{D N}{D N_{i}}=\frac{Q N}{Q_{i} N_{i}} \underset{\text { we denote }}{=} \delta$, and hence $D Q_{i}=\frac{D Q}{\delta}$ and $Q_{i} N_{i}=\frac{Q N}{\delta}$.

Therefore, for side $A B$ there holds:

$$
A B=A P_{i}+P_{i} B=\alpha \cdot A P+\frac{B P}{\beta}=\alpha \cdot \frac{A B}{2}+\frac{A B}{2 \beta}=\frac{1}{2} A B\left(\alpha+\frac{1}{\beta}\right),
$$

or in the other words, $A B=\frac{1}{2} A B\left(\alpha+\frac{1}{\beta}\right)$, and hence $\alpha+\frac{1}{\beta}=2$.

In a similar manner, for side $C D$ there holds:

$$
C D=C Q_{i}+Q_{i} D=\gamma \cdot C Q+\frac{D Q}{\delta}=\gamma \cdot \frac{C D}{2}+\frac{C D}{2 \delta}=\frac{1}{2} C D\left(\gamma+\frac{1}{\delta}\right),
$$

or in the other words, $C D=\frac{1}{2} C D\left(\gamma+\frac{1}{\delta}\right)$, and hence $\gamma+\frac{1}{\delta}=2$.

Therefore, perimeter $P_{P_{i} M_{i} Q_{i} N_{i}}$ can be expressed as follows:

$$
P_{P_{i} M_{i} Q_{i} N_{i}}=P_{i} M_{i}+M_{i} Q_{i}+Q_{i} N_{i}+N_{i} P_{i}=\alpha \cdot N P+\frac{P M}{\beta}+\gamma \cdot M Q+\frac{Q N}{\delta} .
$$

Since circle $\omega_{E F}$ is coordinated with the Pascal points $P$ and $Q$ that are formed by it (see Theorem 3), it follows that quadrilateral $P M Q N$ is a kite in which $N P=P M$ and $N Q=Q M$ (see General Theorem C).

Therefore: $P_{P_{i} M_{i} Q_{i} N_{i}}=N P\left(\alpha+\frac{1}{\beta}\right)+M Q\left(\gamma+\frac{1}{\delta}\right)=2 N P+2 M Q=P_{P M Q N}$. 


\section{Theorem 5.}

Let $A B C D$ be an inscribable quadrilateral in which the diagonals intersect at point $E$, and the continuations of sides $B C$ and $A D$ intersect at point $F$;

$\omega_{i}$ is an arbitrary circle that intersects sides $B C$ and $A D$ at points $M_{i}$ and $N_{i}$, respectively, and which forms Pascal points $P_{i}$ and $Q_{i}$ on sides $A B$ and $C D$, respectively.

Of all the quadrilaterals $P_{i} M_{i} Q_{i} N_{i}$ inscribed in quadrilateral $A B C D$ and defined by circles $\omega_{i}$, the quadrilateral with the maximal area is the one defined by circle $\omega_{E F}$ (a circle whose diameter is the segment EF).

\section{Proof.}

Let $P M Q N$ be a quadrilateral defined by circle $\omega_{E F}$. Let us prove that for any quadrilateral $P_{i} M_{i} Q_{i} N_{i}$ defined by circle $\omega_{i}$, there holds $S_{P_{i} M_{i} Q_{i} N_{i}}<S_{P M Q N}$.

In the proof of Theorem 4, we saw that the corresponding sides of quadrilaterals $P M Q N$ and $P_{i} M_{i} Q_{i} N_{i}$ are parallel to each other. Therefore, in these quadrilaterals, the corresponding angles are equal, in other words: $\measuredangle P=\measuredangle P_{i}, \measuredangle M=\measuredangle M_{i}, \measuredangle Q=\measuredangle Q_{i}$, and $\measuredangle N=\measuredangle N_{i}$.

The quadrilateral $P M Q N$ is a kite, therefore opposite angles $\measuredangle M$ and $\measuredangle N$ are equal. Thus, in quadrilateral $P_{i} M_{i} Q_{i} N_{i}$, angles $\measuredangle M_{i}$ and $\measuredangle N_{i}$ are equal. We denote each of these four angles by $\alpha$ (see Figure 12). 


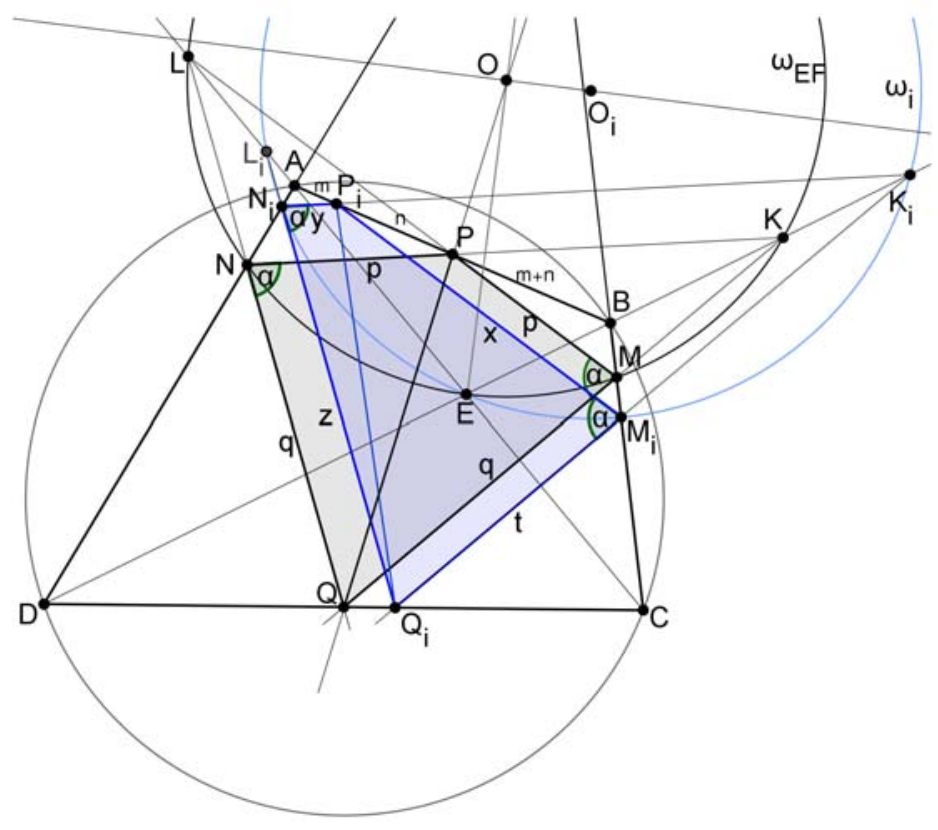

Figure 12.

We denote the sides of kite $P M Q N$ as follows: $P M=P N=p$, $Q M=Q N=q$. We denote the sides of quadrilateral $P_{i} M_{i} Q_{i} N_{i}$ as follows: $P_{i} M_{i}=x, P_{i} N_{i}=y, Q_{i} N_{i}=z$, and $Q_{i} M_{i}=t$.

We denote the segments of side $A B$ as follows: $A P_{i}=m$ and $P_{i} P=n$, and hence $B P=m+n$ (because $P$ is the middle of $A B$ ), $B P_{i}=m+2 n$.

In Theorem 1, we proved that $\frac{B M}{M C}=\frac{A N}{N D}$. We denote each of the ratios that appear in the equality by $\lambda$.

Let us calculate the area of kite $P M Q N$ :

$$
S_{P M Q N}=S_{\triangle P M Q}+S_{\triangle P N Q}=2 \cdot \frac{1}{2} p q \sin \alpha=p q \sin \alpha .
$$

Therefore there holds: $S_{P M Q N}=p q \sin \alpha(*)$. 
THE THEORY OF AN INSCRIBABLE QUADRILATERAL ... 105

In a similar manner, we calculate the area of quadrilateral $P_{i} M_{i} Q_{i} N_{i}$ :

$S_{P_{i} M_{i} Q_{i} N_{i}}=S_{\triangle P_{i} M_{i} Q_{i}}+S_{\Delta P_{i} N_{i} Q_{i}}=\frac{1}{2} x t \sin \alpha+\frac{1}{2} y z \sin \alpha=\frac{1}{2}(x t+y z) \sin \alpha$,

therefore there holds: $S_{P_{i} M_{i} Q_{i} N_{i}}=\frac{1}{2}(x t+y z) \sin \alpha(* *)$.

Now, let us express the lengths of segments $x, y, z$, and $t$ using the lengths of segments $p$ and $q$.

Since $P N \| P_{i} N_{i}$, from the extended Thales' theorem, there holds

$$
\frac{A P_{i}}{A P}=\frac{A N_{i}}{A N}=\frac{P_{i} N_{i}}{P N}(\mathrm{I})
$$

Using our notation defined above:

From the proportion $\frac{A P_{i}}{A P}=\frac{P_{i} N_{i}}{P N}$, it follows that $\frac{y}{p}=\frac{m}{m+n}$, and hence: $y=\frac{m}{m+n} p$.

From the proportion $\frac{A N_{i}}{A N}=\frac{P_{i} N_{i}}{P N}$, it follows that:

$1-\frac{A N_{i}}{A N}=1-\frac{m}{m+n} \Rightarrow \frac{N N_{i}}{A N}=\frac{n}{m+n}$, and hence: $N N_{i}=\frac{n}{m+n} A N(\mathrm{II})$.

From the fact that $P M \| P_{i} M_{i}$, we obtain that $\frac{B P}{B P_{i}}=\frac{B M}{B M_{i}}=\frac{P M}{P_{i} M_{i}}$ (III).

Similarly, from the proportion $\frac{B P}{B P_{i}}=\frac{P M}{P_{i} M_{i}}$, it follows that: $\frac{p}{x}=\frac{m+n}{m+2 n}$, and hence: $x=\frac{m+2 n}{m+n} p$.

From the proportion $\frac{B M_{i}}{B M}=\frac{P_{i} M_{i}}{P M}$, it follows that:

$$
\begin{gathered}
\frac{B M_{i}}{B M}-1=\frac{m+2 n}{m+n}-1 \Rightarrow \frac{M M_{i}}{B M}=\frac{n}{m+n}, \text { and hence: } \\
M M_{i}=\frac{n}{m+n} B M(\mathrm{IV}) .
\end{gathered}
$$


From the fact that $Q M \| Q_{i} M_{i}$, we obtain the proportion: $\frac{C M_{i}}{C M}=\frac{M_{i} Q_{i}}{M Q}$. Based on the above notation, on the last proportion and the fact that $C M_{i}=C M-M M_{i}$, we obtain: $\frac{C M-M M_{i}}{C M}=\frac{t}{q} \Rightarrow 1-\frac{M M_{i}}{C M}=\frac{t}{q}$, and hence, from formula (IV), we have: $1-\frac{n}{m+n} \cdot \frac{B M}{M C}=\frac{t}{q}$, and finally: $t=\left(1-\frac{n}{m+n} \cdot \lambda\right) q$

From the fact that $Q N \| Q_{i} N_{i}$, we obtain

$$
\frac{D N}{D N_{i}}=\frac{N Q}{N_{i} Q_{i}} \Rightarrow \frac{D N}{D N+N N_{i}}=\frac{q}{z} \Rightarrow \frac{1}{1+\frac{N N_{i}}{D N}}=\frac{q}{z}
$$

and hence, from formula (II), we have: $\frac{1}{1+\frac{n}{m+n} \cdot \frac{A N}{N D}}=\frac{q}{z}$, and finally: $z=\left(1+\frac{n}{m+n} \cdot \lambda\right) q$

Now we substitute the expressions for the values $x, y, z$, and $t$, we obtained above in formula $(* *)$, and obtain:

$$
\begin{aligned}
S_{P_{i} M_{i} Q_{i} N_{i}} & =\frac{1}{2}\left(\frac{m+2 n}{m+n} p\left(1-\frac{n}{m+n} \cdot \lambda\right) q+\frac{m}{m+n} p\left(1+\frac{n}{m+n} \cdot \lambda\right) q\right) \sin \alpha \\
& =\frac{1}{2} p q\left(\frac{m+2 n}{m+n}-\frac{m n+2 n^{2}}{(m+n)^{2}} \cdot \lambda+\frac{m}{m+n}+\frac{m n}{(m+n)^{2}} \cdot \lambda\right) \sin \alpha \\
& =\frac{1}{2} p q\left(2-\frac{2 n^{2}}{(m+n)^{2}} \cdot \lambda\right) \sin \alpha=p q\left(1-\frac{n^{2}}{(m+n)^{2}} \cdot \lambda\right) \sin \alpha
\end{aligned}
$$

Or, in the other words: $S_{P_{i} M_{i} Q_{i} N_{i}}=p q\left(1-\frac{n^{2}}{(m+n)^{2}} \cdot \lambda\right) \sin \alpha$. 
Since there holds $0<\left(\frac{A N}{N D}=\lambda\right)<1$, and also $0<\frac{n^{2}}{(m+n)^{2}}<1$, we obtain: $0<\left(1-\frac{n^{2}}{(m+n)^{2}} \cdot \lambda\right)<1$, and therefore:

$$
S_{P_{i} M_{i} Q_{i} N_{i}}=p q\left(1-\frac{n^{2}}{(m+n)^{2}} \cdot \lambda\right) \sin \alpha<p q \sin \alpha=S_{P M Q N}
$$

\section{References}

[1] H. S. M. Coxeter and S. L. Greitzer, Geometry revisited, Maa 19 (1967).

[2] D. Fraivert, The theory of a convex quadrilateral and a circle that forms "Pascal points" - the properties of "Pascal points" on the sides of a convex quadrilateral, Journal of Mathematical Sciences: Advances and Applications 40 (2016), 1-34.

$$
\text { http://dx.doi.org/10.18642/jmsaa_7100121666 }
$$

[3] D. Fraivert, Discovering new geometric properties by spiral inductive deductive investigation, Far East Journal of Mathematical Education, Pushpa Publishing House 16(2) (2016), 185-202.

http://dx.doi.org/10.17654/ME016020185

[4] J. Hadamard, Lessons in Geometry, American Mathematical Society, Vol. I, Plane Geometry, 2008.

[5] Z. Skopets, Geometrical Miniature, Ed. G. D. Gleizer, (In Russian), Prosveshenie, Moscow, 1990.

[6] I. M. Yaglom, Complex Numbers in Geometry, Academic Press, New York, 1968. 